\title{
GAMMA-RAY BURST HOST GALAXY GAS AND DUST*
}

\author{
RHAANA STARLING, RALPH WIJERS AND KLAAS WIERSEMA \\ University of Amsterdam \\ Kruislaan 403, 1098 SJ Amsterdam, The Netherlands \\ rlcs1@star.le.ac.uk;rwijers@science.uva.nl ; kwrsema@science.uva.nl
}

\begin{abstract}
We report on the results of a study to obtain limits on the absorbing columns towards an initial sample of 10 long Gamma-Ray Bursts observed with BeppoSAX, using a new approach to SED fitting to nIR, optical and X-ray afterglow data, in count space and including the effects of metallicity. When testing MW, LMC and SMC extinction laws we find that SMC-like extinction provides the best fit in most cases. A MW-like extinction curve is not preferred for any of these sources, largely since the $2175 \AA$ bump, in principle detectable in all these afterglows, is not present in the data. We rule out an SMClike gas-to-dust ratio or lower value for 4 of the hosts analysed here (assuming SMC metallicity and extinction law) whilst the remainder of the sample have too large an error to discriminate. We provide an accurate estimate of the line-of-sight extinction, improving upon the uncertainties for the majority of the extinction measurements made in previous studies of this sample.
\end{abstract}

\section{Introduction}

The accurate localisation of Gamma-Ray Bursts (GRBs) through their optical and $\mathrm{X}$-ray afterglows has enabled detailed studies of their environments. Selection solely by the unobscured gamma-ray flash has allowed the discovery of a unique sample of galaxies spanning a very wide range of redshifts from $z \sim 0.009$ to $6.3{ }^{3}$ Hence, detailed and extensive host galaxy observations provide a wealth of information on the properties of star-forming galaxies throughout cosmological history.

Afterglow spectroscopy and/or photometry can be used to provide an estimate of the total extinction along the line-of-sight to the GRB. Absorption within our own Galaxy along a particular line of sight can be estimated and removed, but absorption which is intrinsic to the GRB host galaxy as a function of wavelength is unknown, and is especially difficult to determine given its dependence on metallicity and the need to distinguish it from that of intervening systems. In general, low amounts of optical extinction are found towards GRBs, unexpected if GRBs are located in dusty star-forming regions, whilst the X-ray spectra reveal a different picture. At X-ray wavelengths we often measure high values for the absorbing columns, where the absorption is caused by metals in both gas and solid phase. ${ }^{11}$ The apparent discrepancy between optical and X-ray extinction resulting in high gas-to-dust ratios in GRB host galaxies (often far higher than for the MW, LMC or SMC, e.g. GRB $020124^{2}$ ) is not satisfactorily explained, though the suggestion that dust destruction can occur via the high energy radiation of the GRB ${ }^{9}$ could possibly account for the discrepancy.

*The authors acknowledge funding from the EU RTN 'Gamma-Ray Bursts: An Enigma and a Tool', support from PPARC, and RS thanks the conference organisers for financial assistance. 
Traditionally the optical and X-ray spectra have been treated seperately in extinction studies. Since the underlying spectrum is likely a synchrotron spectrum (power law or broken power law) extending through both wavelength regimes, it is most accurate to perform simultaneous fits. We perform simultaneous broadband fits of the spectral energy distributions (SEDs) in count space, so we need not first assume a model for the X-ray spectrum. Inclusion of nIR data and $R$ band optical data together with the $2-10 \mathrm{keV}$ X-ray data, regions over which extinction has the least effect, allows the underlying power law slope to be most accurately determined. This sample of 10 long GRBs observed with the BeppoSAX Narrow Field Instruments is chosen for the good availability (3 bands or more) of optical/nIR photometry.

\section{Results and Discussion}

Detailed results of fits to the SEDs for all GRBs in the sample, and further references, can be found in Starling et al. (2007). Figure 1 shows a comparison of the absorption measurements with Galactic, LMC and SMC gas-to-dust ratios. This plot has been constructed in a number of previous works ${ }^{1846}$ and here we show the observed distribution of $E(B-V)$ and $N_{\mathrm{H}}$ for the first time derived simultaneously from a fit to X-ray, optical and nIR data. We find a large excess in absorption above the Galactic values in two sources: GRBs $000926(E(B-V)$ only) and 010222 , whilst no significant intrinsic absorption is necessary in GRBs 970228 and 990510. The cooling break can be located in three of the afterglows: GRBs 990123,990510 and 010222 and to all other SEDs a single power law is an adequate fit.

We find a wide spread in central values for the gas-to-dust ratios, and for 4 GRBs the gas-to-dust ratios are formally inconsistent with (several orders of magnitude higher than) MW, LMC and SMC values at the $90 \%$ confidence limit assuming the SMC metallicity. This must mean that either gas-to-dust ratios in galaxies can span a far larger range than thought from the study of local galaxies, or the ratios are disproportionate in GRB hosts because the dust is destroyed by some mechanisms (likely the GRB jet), or that the lines of sight we probe through GRBs tend to be very gas-rich or dust-poor compared with random lines of sight through galaxies. A dust grain size distribution which is markedly different than considered here may also affect these ratios.

We have compared the results of this method to those of other methods of determining $E(B-V)$. In particular we find that with respect to continuum fitting methods such as this, optical extinction is overestimated with the depletion pattern method, ${ }^{5}$ and we have quantified this for a small number of cases. ${ }^{7}$ We note, however, that since this is a line-of-sight method, the measured columns may not be representative of the host galaxy as a whole, therefore comparison with the integrated host galaxy methods is important.

Swift, robotic telescopes and Rapid Response Mode on large telescopes such as the William Herschel Telescope and the Very Large Telescopes now allow early, high 
quality data to be obtained, which will help immensely in discriminating between the different extinction laws at work in the host galaxies.

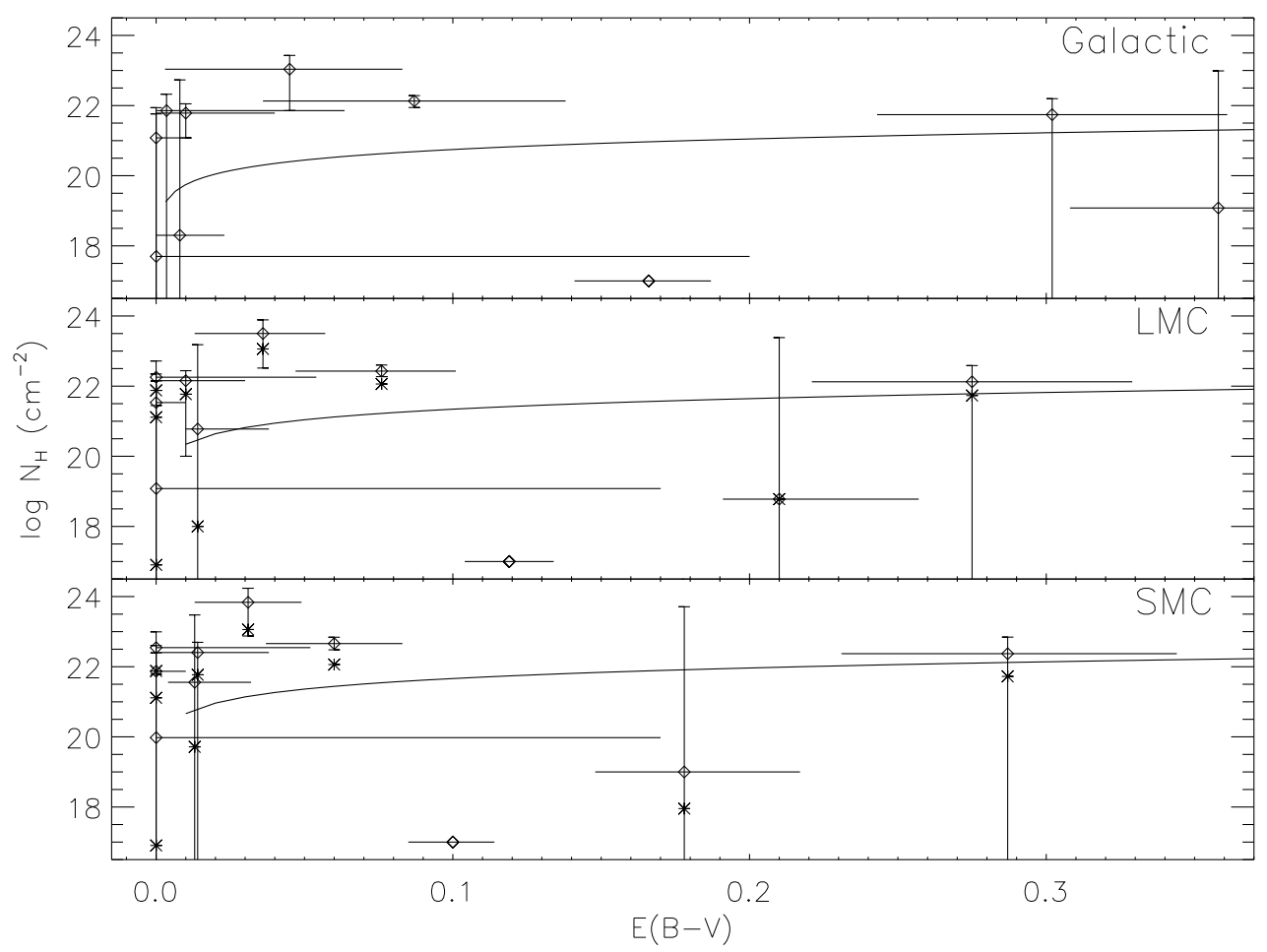

Fig. 1. Intrinsic absorption in optical/nIR $(E(B-V))$ and X-rays $\left(\log N_{\mathrm{H}}\right)$ measured for the GRB sample with $90 \%$ error bars. We compare these with three different optical extinction laws overlaid with solid curves: Galactic (top panel), LMC (middle panel) and SMC (lower panel). Appropriate metallicities are adopted for $\operatorname{LMC}\left(1 / 3 \mathrm{Z}_{\odot}\right)$ and SMC $\left(1 / 8 \mathrm{Z}_{\odot}\right)$ calculations (diamonds), and stars mark the centroids of the Solar metallicity fits. For GRB 000926 the data were too sparse to fit for $N_{\mathrm{H}}$, so we plot the $E(B-V)$ range at $\log N_{\mathrm{H}}=17.0$ for clarity.

\section{References}

1. Galama T. J. and Wijers R. A. M. J., 2001, ApJ, 549, L209

2. Hjorth J. et al., 2003, ApJ, 597, 699

3. Jakobsson P. et al., 2006, A\&A, 447, 897

4. Kann D. A., Klose S. and Zeh A., 2006, ApJ, 641, 993

5. Savaglio S., Fall S. M. and Fiore F., 2003, ApJ, 585, 638

6. Schady P. et al., MNRAS submitted

7. Starling R. L. C. et al., ApJ in press, astro-ph/0610899

8. Stratta G. et al., 2004, ApJ, 608, 846

9. Waxman E. and Draine B. T., 2000, ApJ, 537, 796

10. Wijers R. A. M. J. and Galama T. J., 1999, ApJ, 523, 177

11. Wilms J., Allen A. and McCray R., 2000, ApJ, 542, 914 